\title{
Research on the Information Communication Model of Information Encounter and Information Institutionalization of Information System Developers
}

\author{
Zhenting $\mathrm{Lu}^{1}$, Xiang $\mathrm{Liu}^{2}$ and Liyuan Shang ${ }^{3}$ \\ 1Jiangxi Industry Polytechnic College, Electronic and Information Engineering Branch, JiangXi \\ Nanchang, 330095 \\ 2Jiangxi Technical College of Manufacturing, Department of Information Engineering, JiangXi \\ Nanchang, 330095
}

3Department of Economics and Management , Jiangxi Technical College of Manufacturing, JiangXi Nanchang, 330095

Keywords: Communication model; System development; Information encounter; Information system.

\begin{abstract}
Information communication is the most important method in the development cycle of information system development project, especially for some large and medium-sized information system development project organizations, due to the large scale of the organization, the people and departments institutions involved are complex. Based on information encountering theory and institutional theory, this paper studies the information communication model of information system developers. It firstly describes the current status of the information communication model of information system developers and then it defines and expounds information encountering theory and institutional theory. From the starting points of the two theories, it carries out the information communication model of information system developers. Finally, the paper carries out the brief connotation of this model and the reasonable suggestions of the optimization of the model.
\end{abstract}

\section{Introduction}

Information communication is the most important method in the development cycle of information system development project. Especially for some large and medium-sized information system development project organizations, due to the large scale of the organization, the people and departments institutions involved are complex, if the information communication method is not efficient or reasonable, or the information communication method cannot carry out effective information communication model for information system development, or the information communication method is lack of complete information, exchange mechanism or system specification, in the current situation where the division of an information system development is more and more clear and the developer team cooperation plays more and more important role, which does obviously not conducive to the efficient development link of the information system[1]. In the process of information system project development, in order to ensure that information system 
developers still keep the same voice after the division of the team, obviously communication is very necessary. Once communication is failure, there is no doubt that in the whole team development project, the disadvantages such as low development efficiency and backward will expose. Apparently, in information system development, the smooth of personnel information communication is the precondition of the project and the key to the success of information system development project. So how to improve the information communication of the system developers to a maximum degree has a direct impact on the success of the project.

\section{Method}

By collecting data, this paper uses the literature method and the demonstration analysis method in order to achieve the rationality. Strictly speaking, the innovation of this article is presented based on the information communication model of the information encountering theory and information institutional theory; simply speaking, it is the information institutionalization of information encounter which provides constructive suggestions and reference to the later information system development projects and makes them can better meet the functional requirements of users. Because so far, the research about the information communication model of information system developers has caused a certain amount of attention both in theory and in practice. However, as a whole, the research is not very detailed. From a practical point of view, while there are some cases, but the real effective solutions to deal with the problems of communication barriers between developers has no special specifications. Most solutions are completely the results of independent design and selection. So this article can combine information encounter and institutionalization to study the information communication model between developers, carry out new communication pattern based on encounter and institutionalization and provide corresponding countermeasures and suggestions, which are the innovation of this paper.

The related problems and theories of information system developers and information communication include definition of information system developers, the definition of information communication and information communication model, the definition of information communication of information system developers. It also expounds the status of the information communication of information system developers, based on which, it puts forward the information communication theory of system developers which provides foundation framework for the further discussion and research of information communication model.

The current situation of information communication of information system developers is that there are many problems in information communication of information system developers, the key reason of which is the communication is not smooth. In the system planning stage, project director does not well understand the needs of users, which makes it impossible to well clear the request of system development, and uses get the wrong feasibility study and system analysis personnel information exchange, which leads to the failure of the next phase of development and then the failure of the entire information system development. In the system analysis phase, the system analyst mainly through the communication with project manager analyzes the organization structure and function correctly. If the information communication is not smooth, the business processing and the data processing will not be cleaned. What's more, the wrong analysis to date also leads to a wrong logic plan of information system development. In system design phase, the above information communication barriers lead to chaos of the overall design scheme of the system and the failure of the subsystem function affect which influences the module design, the database design, the input and 
output interface design and the coding design. In short, information communication failure will not be able to provide the correct programming instructions for the next phase. In system implementation stage, the main tasks are coding, testing, system debugging, etc., which put the design of the system into practice and turn the logical model into physical model. The poor information communication will lead to the purchase during the system implementation stage and the installation and commissioning of the system of the information system. And in practice, there are many failures in all stages of system development due to this kind of poor communication. As a result, based on the current information situation of information system developers, to build an optimal information communication model of the information system developers is imperative. This is also the significance of the information communication model of information system developers based on the information encounter and institutionalization. The information communication between information system developers plays an important role in the success of a project. In the development projects of large and medium-sized enterprises, because of the different organizational structure, there may be functional organization structure, matrix organizational structure, linear structure, etc., as a result, when to establish development project team, if it is a matrix structure, namely the developers are from different departments and form temporary project development group, so although sometimes the information communication can be through video conferences or face-to-face communication, due to some environmental impact and the difference of quality level and knowledge ability of each developer, the information communication may be not very smooth. Therefore, a kind of effective information communication model is needed to solve these problems effectively. And the information communication channels of many development projects are single or imperfect which are lack of flexibility, making the information transfer process between the in-house system developers be slow and seriously influencing the process and efficiency of the process of the project development. Therefore, to establish perfect information communication system is helpful to improve the sense of belonging among members to better realize the overall goal of the project development tasks. In the construction of enterprise internal communication system, through the analysis of the disadvantages of the information communication of the enterprises of our country, Qiao Yunli puts forward the preliminary scheme of building information system, determines the goal of information communication, builds up the encouraging communication range and establishes the internal communication supervision and evaluation mechanism. The poor information communication is one of the main reasons of the failure of IT projects. As a result, the smooth of information communication plays an important role in the development of enterprises and the development of the information development, since information communication is always the main body of existence. Although the research of the information communication ability in the doctor-patient information system development projects which does not belong the same field, it is also a branch of information system. Theoretically, the experience can be referred to the research of the communication in other general information system development projects.

Currently in the process of information system development, because both the traditional information communication model and the more complex information communication model have their own advantages and disadvantages, as it is mentioned above, the information gained by the information encounter is not the information which information system developers consciously searches for, namely the information is even not known by information system developers but is just encountered. The information inspires developers and makes them realize this kind of information have great relationship with the information system development, such as the information which is 
encountered in information encountering environment, such as the everyday life and working environment of toilet, Weibo, Weichat, QQ group, Rrenren or MSN. As mentioned above, the information has big help for the development of information system. So, developers should make an effort to institutionalize and normalize the information which is gained by this kind of information encounter in order to make the information communication of information system developers based on information encountering and institutional pattern be more standardizing, specific and hierarchical in order to greatly improve the efficiency of information system development. According to the information communication of the information system developers, the paper puts forward a model which is information communication model of the information system developer based on information encountering and institutional pattern.

In the development of planning, analysis, design and implementation phase of the information system, the information communication between information system developers is throughout the whole process. The course of the logic model to the physical model is the process of the various information communication system developers. Regardless of different types of information communication, such as wheel type, Y type, along with all channel, whole single, single string type, the quality of the information communication is directly related to the efficiency of information system development and the quality of information system. The accuracy of information communication can ensure to convert existing artificial information management mode to modern information management model with the use of computer technology. Information exchanges can contribute to the formation of human resources. Information communication in the process of information system development is endless, and the system developers involved in the process of information system development process is a distributed group. To ensure the stability of the members of the development project group, a good interaction is needed. Although all the members will participate in the control and learning of the information system development, it is not enough to just rely on the kind of formal unification and study. The information of information system developers gained by information encounter plays a critical role in the expansion of the thinking of the information system developers. So it is reasonable to ensure the continuous developing in the process of interaction of this kind of dynamic information communication and sharing. However, the information communication only through the unified form of the meeting or the unified form of learning between members of the system development is not enough to provide complete information or become the only provider of information. The information gained in the information encounter environment is converted, transferred and shared by people in different information media in order to achieve the system development of knowledge sharing. Under this situation, due to the wide range of liquidity of people, the openness and interactivity of networks without the limitation of space and time, the information communication is not very smooth. As a result, it is necessary to make institutionalization and standardization of the information communication in the environment of information encounter in information system development, based on which, according to the related paper of the field, this paper puts forward a pattern, which is a information communication model of information system developers based on information encounter and information institutionalization, in order to make the information gained in the environment of information encounter can be more standardized, hierarchical and special in the pattern of this paper and to greatly improve the efficiency and quality of information system development.

Information system development requires the joint efforts of a project team, in which each member must keep coordination to make the whole project smooth[26]. In planning, analysis, design 
and implementation phase of the information system, the information communication of any every two or more members of the system development has a certain influence on the development of information system. Only the information communication is unobstructed can make the recessive knowledge be explicit, as well as to enable participants to share or access to information. Based on the pattern and the information encounter and information institutionalization, the paper puts forward the following suggestions:

Form and specify information encounter community groups of information system developers.

Develop and institutionalize the environment of information encounter of information system developers.

Strengthen and perfect the safety and maintenance of information encounter environment.

Deepen the information content community filtering of information encounter community groups.

Optimize information communication of information system developers with information encountering and institutionalized pattern.

\section{Conclusions}

This paper firstly summaries the research background and significance of the topic and briefly introduces the research status of this thesis. This paper belongs to the problem which is worth studying in the field of information science and should be conduct further study based on the former research results. Then, it summaries the related problems of the information communication system developers and the information communication. Information system development period can be basically divided into the planning of system, the analysis of system, the design of system, the implementation of system, the maintenance and evaluation of system. But in this paper, the information system development is defined as the stage from the user demand analysis to the stage before the system's online implementing, which does not involve system maintenance. Then the paper again defines the information system development involved in this paper which is especially large and medium-sized information system development project. It also again defines the information system developers as the information competent $\mathrm{CIO}$, the project mana ger, the end user, the systems analyst, the system designer, the program designer, the tester, and it defines the information communication and information communication model. Then it analyzes the current situation of the information communication of information system developers and the realistic significance of studying the information communication model of information system developers. Based on the concept and the environment of information encounter, the paper analyzes the relationship between information encounter and the information communication, and explains the principle of institutionalization and the relationship between institutionalization and information system and then it explores the connection between information encounter and information institutionalization. From the internal mechanism of the information communication law between information system developers, during the information system process, it carries out information communication model between information system developers based on information encounter and information institutionalization. Finally, the paper puts forward suggestions on the information communication model between information system developers which optimizes the information communication between information system developers based on information encounter and information institutionalization pattern, makes the information communication have a better effect, and is advantageous to information acquisition of information encounter between information system 
developers, in order to reduce development costs and better complete information system development tasks.

\section{References}

[1] Orlikowski W J, Robey D. Information technology and the structuring of organizations[J]. Information systems research, 1991, 2(2): 143-169.

[2] Lamb R, Kling R. Reconceptualizing users as social actors in information systems research[J]. MIS quarterly, 2003: 197-236.

[3] Stein E W, Zwass V. Actualizing organizational memory with information systems[J]. Information systems research, 1995, 6(2): 85-117.

[4] Davenport T H, Short J. Information technology and business process redesign[J]. Operations management: critical perspectives on business and management, 2003, 1: 97.

[5] Avgerou C. Information systems in developing countries: a critical research review[J]. Journal of information Technology, 2008, 23(3): 133-146.

[6] Jarvenpaa S L, Staples D S. The use of collaborative electronic media for information sharing: an exploratory study of determinants[J]. The Journal of Strategic Information Systems, 2000, 9(2): 129-154.

[7] Nandhakumar J, Avison D E. The fiction of methodological development: a field study of information systems development[J]. Information technology \& people, 1999, 12(2): 176-191.

[8] Dewett T, Jones G R. The role of information technology in the organization: a review, model, and assessment[J]. Journal of management, 2001, 27(3): 313-346.

[9] Lyytinen K, Robey D. Learning failure in information systems development[J]. Information Systems Journal, 1999, 9(2): 85-101.

[10]Newman M, Robey D. A social process model of user-analyst relationships[J]. Mis Quarterly, 1992: 249-266. 